\title{
EXTENDED STAR FORMATION IN THE INTERMEDIATE-AGE LARGE MAGELLANIC CLOUD STAR CLUSTER NGC 2209
}

\author{
Stefan C. Keller, A. Dougal Mackey, and Gary S. Da Costa \\ Research School of Astronomy and Astrophysics, Australian National University, Canberra, Australia \\ Received 2012 August 9; accepted 2012 October 22; published 2012 November 15
}

\begin{abstract}
We present observations of the 1 Gyr old star cluster NGC 2209 in the Large Magellanic Cloud made with the GMOS imager on the Gemini South Telescope. These observations show that the cluster exhibits a main-sequence turnoff that spans a broader range in luminosity than can be explained by a single-aged stellar population. This places NGC 2209 amongst a growing list of intermediate-age (1-3 Gyr) clusters that show evidence for extended or multiple epochs of star formation of between 50 and $460 \mathrm{Myr}$ in extent. The extended main-sequence turnoff observed in NGC 2209 is a confirmation of the prediction in Keller et al. made on the basis of the cluster's large core radius. We propose that secondary star formation is a defining feature of the evolution of massive star clusters. Dissolution of lower mass clusters through evaporation results in only clusters that have experienced secondary star formation surviving for a Hubble time, thus providing a natural connection between the extended main-sequence turnoff phenomenon and the ubiquitous light-element abundance ranges seen in the ancient Galactic globular clusters.
\end{abstract}

Key words: globular clusters: general - Magellanic Clouds

Online-only material: color figure

\section{INTRODUCTION}

Globular clusters (GCs) were once thought to embody simple stellar populations. Their stars of defined distance have appeared coeval and monometallic in composition. This has given GCs a vital role in developing our understanding of the stellar evolution of low-mass and low-metallicity stars. However, with closer inspection, we now know that GCs are not simple systems, but rather exhibit a variety of complex phenomena. The GCs of the Milky Way show a characteristic chemical signature, namely, strong $\mathrm{CN}-\mathrm{CH}, \mathrm{O}-\mathrm{Na}$, and $\mathrm{Mg}-\mathrm{Al}$ anti-correlations (see Gratton et al. 2012 for a review). These abundance variations are rarely seen among field stars of similar age and metallicity (Martell et al. 2011) and extend from the red giant branch (RGB) to the main sequence. This implies that the variations are not due to evolutionary mixing within the stars, but rather some primordial process in the chemodynamical evolution of GCs. Photometric evidence for the presence of multiple stellar populations in ancient GCs has grown in recent years, initially from the most massive systems and recently in GCs of more average mass. This evidence includes the presence of multiple main-sequence, sub-giant, and red giant branches (see Gratton et al. 2012 for a review).

These observed properties pose serious challenges for models of GC formation and evolution. The O-Na anti-correlation calls for material processed via $p$-capture reactions at high temperatures (Denisenkov \& Denisenkova 1989; Langer et al. 1993). The dominant paradigm is that material cycled within asymptotic giant branch stars (AGBs) and subsequently lost in winds, or winds from rapidly rotating massive stars pollute a reservoir of gas from which subsequent star formation takes place. In scenarios where multiple episodes of star formation occur in situ, it is not clear how GCs could retain sufficient gas for timescales of the order of $100 \mathrm{Myr}$ in order to form a second generation (SG) of stars in the face of SNII feedback (Carretta et al. 2010), or how the retained gas could obtain the re- quired composition (e.g., significant helium enhancement in the O-poor, Na-rich stars in some clusters).

\subsection{New Insights from the Intermediate-age Clusters of the Large Magellanic Cloud}

The GCs of the Milky Way are, without exception, ancient populations and do not afford direct insight into the chemodynamical evolution of massive stellar clusters. The Large Magellanic Cloud (LMC), on the other hand, possesses many clusters with masses similar to those of typical Galactic GCs, but spanning a wide range of ages: $\sim 10^{6}$ to $10^{10} \mathrm{yr}$.

Using HST/ACS imaging, Mackey \& Broby Nielsen (2007) showed that the LMC cluster NGC $1846\left(M \sim 10^{5} M_{\odot}\right.$; age $\sim 1.8 \mathrm{Gyr} ;[\mathrm{Fe} / \mathrm{H}] \sim-0.4$ ) possesses a color-magnitude diagram (CMD) exhibiting two distinct main-sequence turnoffs (MSTOs). The width of the RGB is small, indicating that there is no significant internal spread in metallicity. With this constraint, two stellar populations with ages $\sim 300 \mathrm{Myr}$ apart can reproduce the observed CMD.

Various additional HST/ACS data revealed another 10 LMC clusters and 1 Small Magellanic Cloud cluster, of ages between $\sim 1-2.5$ Gyr, that exhibit unusual MSTOs (Mackey et al. 2008a; Glatt et al. 2008; Milone et al. 2009; Rubele et al. 2011; Goudfrooij et al. 2011a, 2011b). The MSTO region of these clusters may be bifurcated or more extended than can be accounted for by photometric errors. Binary stars add confusion around the MSTO but cannot alone explain the peculiar MSTOs (Milone et al. 2009; Goudfrooij et al. 2011b; Yang et al. 2011), similarly stellar rotation appears an unlikely solution (Girardi et al. 2011). Milone et al. report that 11 of 16 (i.e., $70 \% \pm 25 \%$ ) intermediate-age clusters of the LMC possess this phenomenon.

In Keller et al. (2011), we have simulated stellar populations with a range of luminosities and star formation histories. Our simulations show that a cluster with a star formation history spanning $300 \mathrm{Myr}$ would be undetectable to existing HST/ACS photometry of LMC clusters once the age of the cluster exceeds 


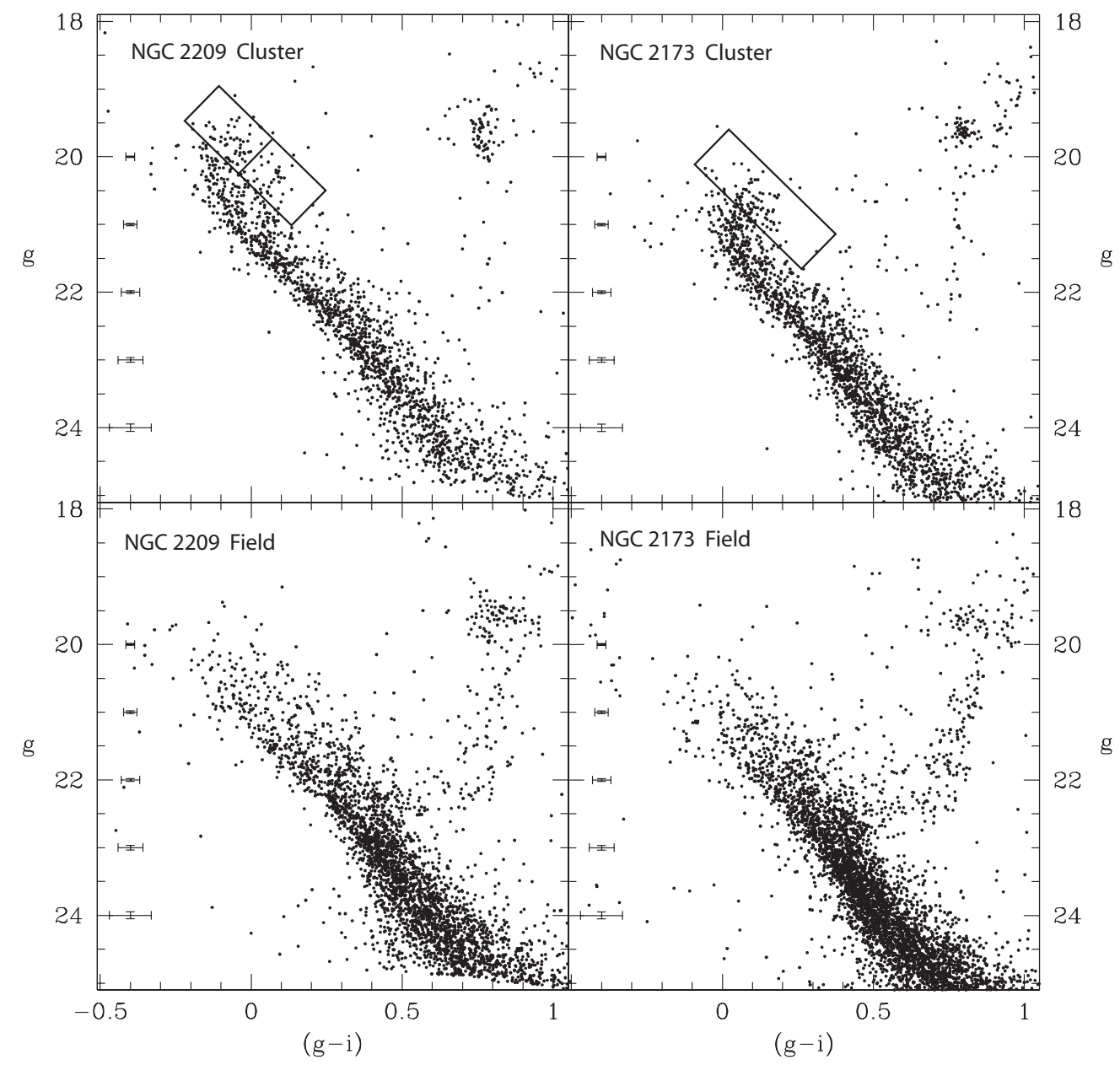

Figure 1. Color-magnitude diagrams derived from GMOS imaging. For NGC 2209 (left), the cluster sample is defined from $40^{\prime \prime}<r<80^{\prime \prime}$ and the field population as $r>125^{\prime \prime}$ from the cluster center. For NGC 2173 (right), the cluster sample is defined from $30^{\prime \prime}<r<70^{\prime \prime}$ from the cluster center and $r>125^{\prime \prime}$ for the field sample. NGC 2209 (left panel) exhibits a main-sequence turnoff morphology that is broader than expected from a single population. Photometric uncertainties are shown by the error bars to the left in each panel. The rectangle shows the selection window for Figure 2.

$\sim 2.3$ Gyr. This is a consequence of the fact that in increasingly older clusters, the difference in age between the constituent stellar populations represents a diminishing fraction of the cluster age, hence the multiple populations become increasingly harder to resolve photometrically. The fact that the extended MSTO phenomenon is only seen in $\sim 1-2.5$ Gyr GCs is therefore not unexpected as this is the window of age from inception of the SG to invisibility. This leads us to claim that the production of multiple populations is an evolutionary phase for the majority of massive clusters. Since less massive clusters evaporate on the timescale of several Gyr, we have asserted a direct connection between the extended MSTO phenomenon and the ubiquitous light element chemical inhomogeneities seen in the ancient GCs.

The currently known multiple MSTO clusters possess another outstanding feature. The magnitude of the age spread exhibited by the stellar population is correlated with the cluster core radius. The most extended clusters for their age possess the largest age spread. This argues that the processes that lead to the formation of multiple stellar populations also impart a dynamical modification to the cluster. The intermediate-age LMC cluster NGC 2209 exhibits a core radius similar to that of NGC 1846, the archetypal multiple MSTO cluster. This led us to predict that the cluster NGC 2209 would exhibit multiple MSTOs (Keller et al. 2011). In this Letter, we describe observations that validate our prediction.

\section{NGC 2209: EXTENDED STAR FORMATION OBSERVED}

As part of an observational study aimed at constructing a census of the occurrence of multiple stellar populations, we have recently obtained GMOS imaging from the Gemini South telescope (GS-2012A-Q-15 and GS-2011A-Q-43). Our sample nearly doubles the number of intermediate-age clusters examined for the presence of multiple stellar populations from 16 to 30. Importantly, our observations provide an age- and luminosity-limited sample of stellar clusters. Our observations are described in detail in a forthcoming paper.

Imaging in $g$ and $i$ was acquired under excellent conditions (IQ 20 and CC 50). This resulted in a seeing of 0.'42-0.'70. The images were photometered using point spread function (PSF) fitting via the Dolphot software package (Dolphin 2000; also used for the artificial star tests). Great care was taken to filter the photometry lists of spurious detections and poor measurements using parameters derived from the PSF fitting. We are able to construct cluster CMDs from the brightest cluster red giants to $\sim 5$ mag below the MSTO. In Figure 1 we show the CMDs for two clusters, NGC 2173 and NGC 2209. Also shown in this figure are the photometric uncertainties determined through artificial star addition to the processed frames. Through artificial star addition we are able to investigate the effects of crowding on the photometry. Crowding becomes sufficiently problematic 


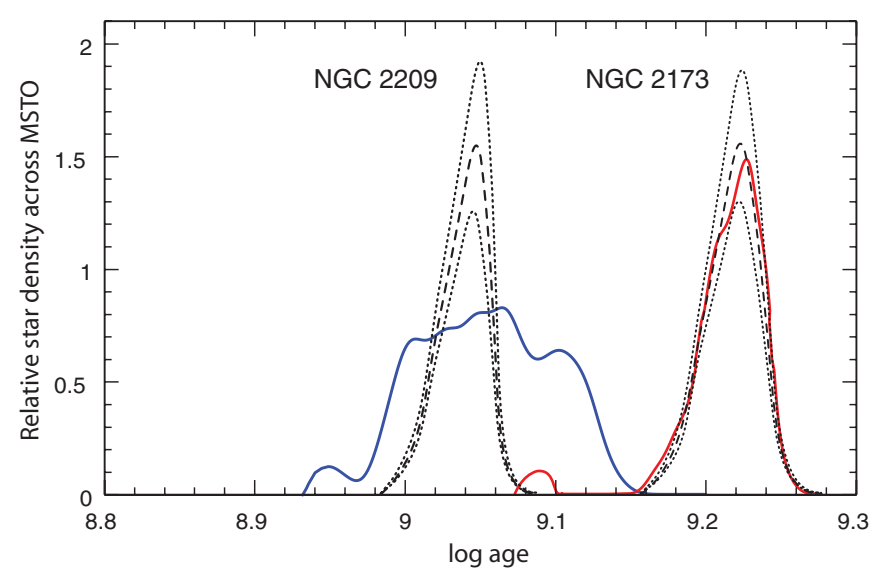

Figure 2. Probability density function of stars in the vicinity of the mainsequence turnoff for NGC 2209 (left) and NGC 2173 (right). Dashed lines show the corresponding distribution from the simulation of a single-age population that includes a $30 \%$ binary fraction and the photometric uncertainties of our observations (upper and lower dotted lines: $1 \sigma$ limits).

(A color version of this figure is available in the online journal.)

in the cluster inner regions, therefore we limit our attention to stars in NGC 2209 in excess of 40" from the cluster center. The outer radius to the cluster sample of $80^{\prime \prime}$ is then set to where the density of stars reaches 1.5 times the density of the field (as determined $>125^{\prime \prime}$ from the cluster center). The cluster sample drawn from this annulus amounts to $13.8 \%$ of the 5.5 square GMOS frame, and the field sample is drawn from the outermost $55 \%$ of the imaged area. In the case of NGC 2173, the cluster sample is defined within 30" $<r<70^{\prime \prime}$ (11.5\% of the imaged area).

In the derived CMD for NGC 2173 we do not see evidence for extended MSTO morphology. The CMD of NGC 2209, on the other hand, shows a pronounced broadening of the MSTO. NGC 2173 is older than NGC 2209 (see below). As discussed in Keller et al. (2011), an older age does make resolution of an extended MSTO more difficult but not invisible in the case of NGC 2173. As well as not being present in the comparison cluster, this broadening at the MSTO is counter to that expected from photometric uncertainties that decrease with decreasing apparent magnitude.

The CMD for the field is dominated by markedly older stellar populations. By considering the ratio of the area covered by the cluster sample to that of the field sample, we determine the field contribution superimposed on the cluster sample. For NGC 2209, between $19.5<g<20.5$ there are expected to be 16 field out of 164 cluster stars (10\%). Similarly, for NGC 2173 the field contributes $6 \%$ to the cluster sample between $20.3<$ $g<21.3$. The inclusion of field stars reduces the contrast of the MSTO, however, they cannot explain the observed dispersion at the cluster MSTO. As discussed extensively in other studies, the superposition of a binary star sequence (Yang et al. 2011) or the effects of stellar rotation (Girardi et al. 2011; Goudfrooij et al. 2011a) are similarly insufficient to account for the MSTO morphology. In the case of both clusters, the reddening is small (see below) and there is no evidence for large differential reddening over the extent of either cluster that could lead to the CMD morphology. Star formation over an extended timeframe or in a series of episodes is, on the other hand, able to explain the flaring of the MSTO.

In Figure 2, we show the probability density function of stars drawn from the rectangle seen in Figure 1 at the MSTO.
Isochrones from Marigo et al. (2008) are used to establish the age of MSTO stars from their position within this region. The isochrones are placed at a distance modulus of 18.54 (Keller \& Wood 2006) and $E(B-V)=0.08$ (Haschke et al. 2012). The age of each star is represented by a Gaussian probability density function of half-width given by the photometric uncertainty established by artificial star addition. Uncertainty in reddening imposes a systematic limitation on the accuracy of age determination of \pm 0.04 in log age. The mean ages for NGC 2173 and NGC 2209 are found to be log age $9.23 \pm 0.05$ and $9.06 \pm 0.05$, respectively. The uncertainties quoted are those relative to our choice of isochrones, systematic uncertainties between models dominate the determination of absolute cluster ages as we discuss below.

Also shown in Figure 2 are the probability density functions of simulated stellar populations without age spread, but with a $30 \%$ binary population and the photometric uncertainties of the observed sample. To generate each model stellar population, we populate the isochrones of Marigo et al. (2008) to achieve the number of stars observed in the range $20.5<g<21.5$ for NGC 2209 and $22<g<23$ for NGC 2173. Uncertainty limits $(1 \sigma)$ above and below the mean are shown based on Poisson statistics of the observed cluster sample within the MSTO region. NGC 2173 shows a histogram that is comparable to that derived from a single-aged stellar population. Following the procedure of Keller et al. (2011), we have experimented with the simulation of CMDs for clusters with a range of internal age spreads. These trials show that, at most, an age difference of 50 Myr could be accommodated by the NGC 2173 data. NGC 2209, on the other hand, shows a distribution that cannot be replicated with a single-aged population. For that matter, the MSTO of NGC 2209 cannot be reproduced by bimodal star formation, but rather shows evidence for extended star formation over a $300 \mathrm{Myr}$ period. In Figure 3, we show a series of isochrones spanning log age 9.0-9.4 at $[\mathrm{Fe} / \mathrm{H}]=-0.4$ (Marigo et al. 2008). We see how a significant age spread is accommodated in the case of NGC 2209, but not for NGC 2173.

Our finding in the case of NGC 2173 for a low ( $<50 \mathrm{Myr}$ ) age range for star formation is contrary to the finding of Bertelli et al. (2003), who found evidence for a $300 \mathrm{Myr}$ age spread. The analysis of Bertelli et al. is based on the photometry in similar seeing to that of the present study. However, the work extracts the cluster sample from between $4^{\prime \prime}<r<140^{\prime \prime}$ from the cluster center. We posit that it is the effect of crowding on the photometry, in particular, within $r<30^{\prime \prime}$ that leads to the interpretation by Bertelli et al. of an age spread.

Dividing the MSTO population in NGC 2209 along a line that bisects the extent in luminosity of the MSTO reveals that $51 \%$ lie on the brighter, and hence younger, branch. This is comparable to that seen in other extended MSTO clusters in the LMC, where Milone et al. (2009) find a typical predominance of $70 \%$ SG stars.

\section{DISCUSSION: IMPLICATIONS FOR THE FORMATION AND EVOLUTION OF MASSIVE STAR CLUSTERS}

In Keller et al. (2011), we show that clusters with extended MSTO morphology have the largest core radii clusters at a given age. This is apparent in Figure 4. Our finding that the cluster NGC 2209 possesses multiple epochs, or an extended epoch, of star formation is a confirmation of this ansatz. To construct this figure, we have not utilized tabulated values from previous works that have utilized a heterogeneous set of stellar evolutionary models. Drawing upon isochrones from a range of 


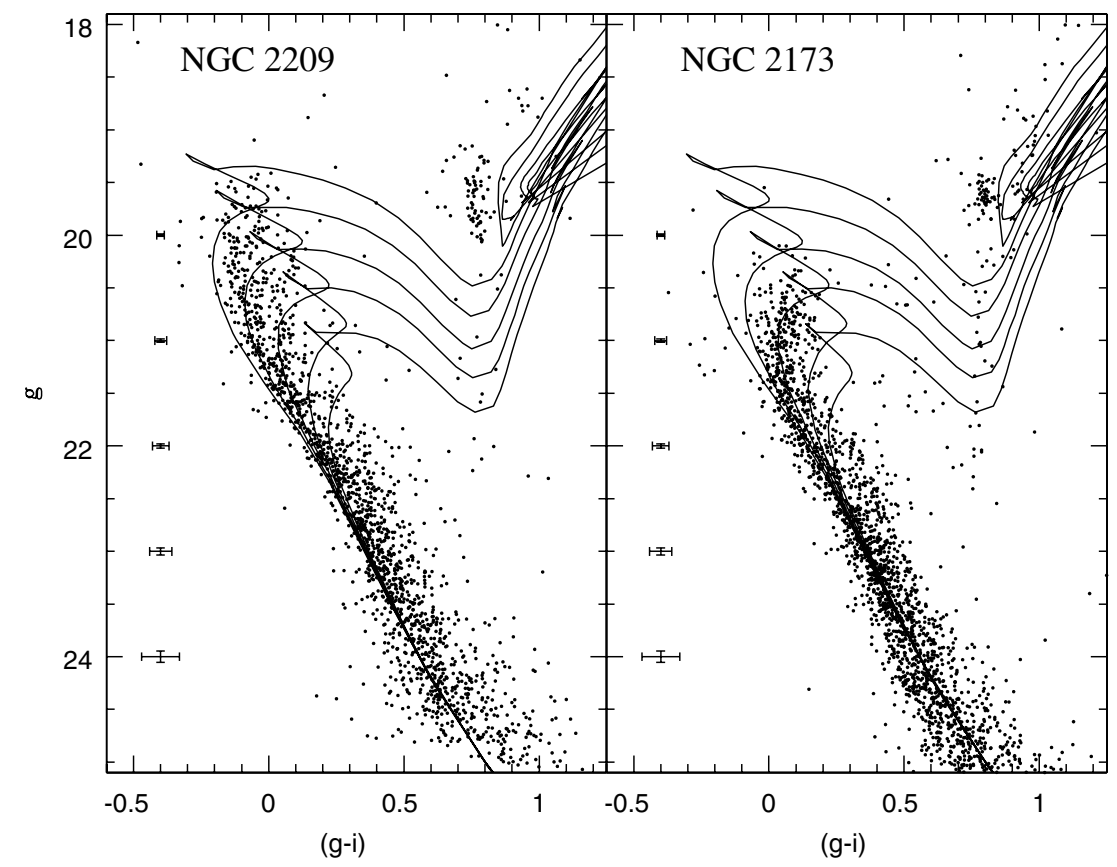

Figure 3. Color-magnitude diagrams of NGC 2209 and NGC 2173 with isochrones for log age 9.0-9.4, $Z=0.008$ from Marigo et al. (2008) overlaid.

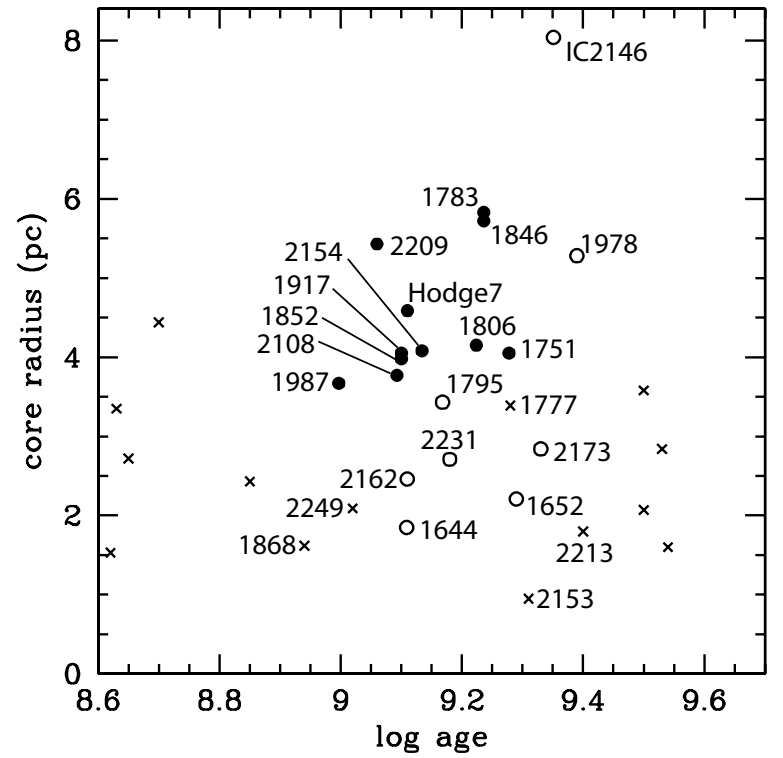

Figure 4. Cluster core radius as a function of cluster age for clusters in the LMC with determined core radii. As discussed in Keller et al. (2011), clusters with extended main-sequence turnoff morphology (solid circles) are without exception those clusters with the largest core radii at a given age. Open symbols show clusters for which studies with sufficient spatial resolution and imaging depth have been conducted but have not shown extended main-sequence turnoff morphology. Crosses are those for which appropriate data do not yet exist.

sources introduces systematic uncertainties in cluster ages of $\sim 40 \%$. Instead, we determine cluster age from the dereddened $g$ (in the case our photometry), or F555W magnitude (from Milone et al. 2009 and Mackey \& Gilmore 2003) of the MSTO (reddening from Haschke et al. 2012; $A_{V}=3.1 E(B-V)$ ) compared to model isochrones of Marigo et al. (2008; again at a distance modulus of 18.54). Thus, we obtain cluster ages that are relative to a known set of isochrones. For clusters with extended MSTO, we have calculated the age at the median magnitude of the MSTO region. Values of $r_{c}$ are taken from Mackey \& Gilmore (2003).
The correspondence between large cluster core radius and the presence of extended MSTO morphology implies that either the formation of stars subsequent to the primary stars of the cluster is fostered by the environment of a cluster of large core radius or it imparts a kinematic signature that acts to increase the core radius of the host cluster. In order to understand the implications of Figure 4, we now briefly review mechanisms of formation and evolution for massive stellar clusters.

The challenge for a scenario of in situ star formation is that which is faced by the formation of multiple populations in the ancient GC population, namely, how a cluster of moderate mass is able to hold on to gas in the face of feedback from supernovae (Gratton et al. 2012). In the context of the ancient GCs, a scenario of gas retention and subsequent star formation is motivated by the apparently ubiquitous presence of light element abundance inhomogeneities in their constituent stars. In this paradigm, gas that has been processed at high temperatures, either within AGB (see, for example, D'Ercole et al. 2012) or massive rapidly rotating stars (Decressin et al. 2009), is retained within the young GC system. The age range exhibited in the extended MSTO clusters of 100-300 Myr (Goudfrooij et al. 2011a) is comparable to that required by AGB star self-pollution.

D'Ercole et al. (2008) present simulations that show that gas ejected by first generation (FG) AGB collects in the cluster core via a cooling flow. The AGB mass loss, plus advected pristine material, is then transformed into an SG of stars. Galactic GCs are known to be composed predominantly of SG stars (Carretta et al. 2010), therefore a large fraction of the initial FG population must be lost. D'Ercole et al. show that such preferential loss of FG stars is enhanced by primordial mass segregation. Within an initially mass-segregated cluster, stellar evolution induced mass loss from massive FG stars removes mass from the inner regions of the cluster. This leads to core expansion and the loss of stars at the outskirts of the cluster (Mackey et al. 2008b). Stars that are lost are primarily FG stars, whereas the SG population remains largely unscathed due to its concentration in the innermost cluster regions. The resulting cluster after a Hubble time exhibits a ratio of SG to FG stars in 
line with observations of Galactic GCs. As shown in Mackey et al. (2008b), primordial mass segregation leads to a cluster that at intermediate age (approximately $9<\log$ age $<9.3$ ) exhibits a larger core radius than unsegregated peers.

In the context of Figure 4, we would therefore expect the intermediate-age clusters with large core radius to be those that exhibit the extended MSTO phase. This is confirmed by observation: intermediate-age clusters with large core radii are those that show extended MSTO morphology. We propose that the extended MSTO phenomenon is a common pathway for massive stellar clusters. The scenario we propose here is in agreement with and extends arguments by previous authors (Keller et al. 2011; Conroy \& Spergel 2011; Goudfrooij et al. 2011a). It ties together the observational constraints from both the extended MSTO clusters and the ancient GC populations in a coherent manner as follows. On the basis of light-element abundance variations, we surmise that GC is composed of FG and SG stars; in extended MSTO clusters we resolve the FG and SG populations on the CMD. The time between FG and SG populations in extended MSTO clusters is between 50 and $460 \mathrm{Myr}$; this is an appropriate time frame for the generation of light-element abundance variations due to the incorporation of processed material in GCs. Observations show that extended MSTO clusters are dominated by younger (SG) stars (the present study; Milone et al. 2009) as are GCs. Goudfrooij et al. (2011a) show that within extended MSTO clusters the SG population is more centrally condensed than the older FG population and furthermore, the higher the cluster mass, the higher the degree of SG concentration in line with expectation from in situ formation (in GCs this signature is lost due to two-body relaxation).

Only clusters of mass similar to, or greater than, those now exhibiting the extended MSTO at an age of 1-3 Gyr $\left(M_{\mathrm{cl}} / M_{\odot} \sim 10^{4}\right.$; Mackey \& Gilmore 2003) possessed a sufficiently deep potential to capture and hold enriched gas for SG formation. Evolution of the star cluster mass function rapidly removes those clusters of lower mass, thus distilling the cluster population over a Hubble time to one that is exclusively massive, and one that is characterized by the chemical signature of multiple populations. A key missing piece of evidence lies in the detailed chemistry of the stars within the intermediate-age clusters. Light-element abundance variations are a prediction of our scenario. To date, only a limited sample of stars have been examined in extended MSTO clusters (Mucciarelli et al. 2009), and these do not show strong evidence for the incorporation of chemically cycled material. Comparison of the detailed chemistry of an expanded set of RGB stars in extended MSTO clusters to nucleosynthetic yields of appropriate metallicity AGB models will be fundamental for placing the extended
MSTO phenomenon in the context of the evolution of massive stellar clusters.

Research by S.C.K. and G.D.C. has been supported by ARC Discovery Project grants DP0878137 and DP120101237. A.D.M. is grateful for support from ARC Australian Research Fellowship DP1093431. Based on observations obtained at the Gemini Observatory (GS-2012A-Q-15, and GS-2011A-Q43), which is operated by the AURA, under a cooperative agreement with the NSF on behalf of the Gemini partnership: the National Science Foundation (United States), the Science and Technology Facilities Council (United Kingdom), the National Research Council (Canada), CONICYT (Chile), the Australian Research Council, Ministério da Ciência, Tecnologia e Inovação (Brazil), and Ministerio de Ciencia, Tecnologia e Innovacin Productiva (Argentina).

\section{REFERENCES}

Bertelli, G., Nasi, E., Girardi, L., et al. 2003, AJ, 125, 770

Carretta, E., Bragaglia, A., Gratton, R. G., et al. 2010, A\&A, 516, 55

Conroy, C., \& Spergel, D. N. 2011, AJ, 726, 36

Decressin, T., Charbonnel, C., Siess, L., et al. 2009, A\&A, 505, 727

Denisenkov, P. A., \& Denisenkova, S. N. 1989, Astronomicheskij Tsirkulyar, 1538,11

D’Ercole, A., D'Antona, F., Carini, R., Vesperini, E., \& Ventura, P. 2012, MNRAS, 423, 1521

D’Ercole, A., Vesperini, E., D’Antona, F., McMillan, S. L. W., \& Recchi, S. 2008, MNRAS, 391, 825

Dolphin, A. E. 2000, PASP, 112, 1383

Girardi, L., Eggenberger, P., \& Miglio, A. 2011, MNRAS, 412, L103

Glatt, K., Grebel, E. K., Sabbi, E., et al. 2008, AJ, 136, 1703

Goudfrooij, P., Puzia, T. H., Chandar, R., \& Kozhurina-Platais, V. 2011a, ApJ, 737,4

Goudfrooij, P., Puzia, T. H., Kozhurina-Platais, V., \& Chandar, R. 2011b, ApJ, 737,3

Gratton, R. G., Carretta, E., \& Bragaglia, A. 2012, A\&AR, 20, 50

Haschke, R., Grebel, E. K., \& Duffau, S. 2012, AJ, 144, 106

Keller, S. C., Mackey, A. D., \& Da Costa, G. S. 2011, ApJ, 731, 22

Keller, S. C., \& Wood, P. R. 2006, ApJ, 642, 834

Langer, G. E., Hoffman, R., \& Sneden, C. 1993, PASP, 105, 301

Mackey, A. D., \& Broby Nielsen, P. 2007, MNRAS, 379, 151

Mackey, A. D., Broby Nielsen, P., Ferguson, A. M. N., \& Richardson, J. C. 2008a, ApJ, 681, L17

Mackey, A. D., \& Gilmore, G. F. 2003, MNRAS, 338, 85

Mackey, A. D., Wilkinson, M. I., Davies, M. B., \& Gilmore, G. F. 2008b, MNRAS, 386, 65

Marigo, P., Girardi, L., Bressan, A., et al. 2008, A\&A, 482, 883

Martell, S. L., Smolinski, J. P., Beers, T. C., \& Grebel, E. K. 2011, A\&A, 534, A136

Milone, A. P., Bedin, L. R., Piotto, G., \& Anderson, J. 2009, A\&A, 497, 755

Mucciarelli, A., Origlia, L., Ferraro, F. R., \& Pancino, E. 2009, ApJ, 695, L134

Rubele, S., Girardi, L., Kozhurina-Platais, V., Goudfrooij, P., \& Kerber, L. 2011, MNRAS, 414, 2204

Yang, W., Meng, X., Bi, S., et al. 2011, ApJ, 731, L37 